\title{
EDITORIAL
}

\section{HOST GENOME INTERACTIONS IN COVID-19: DIFFICULTIES IN PATIENT MANAGEMENT AND HEALTHCARE POLICY MANAGEMENT}

1. Mankind during current pandemic has displayed the best possible responses utilizing researched knowledge base, cutting edge biotechnology and most importantly the will to take down this mammoth menace of our times. Though mixed sorrows and failures progress towards the "war on COVID-19" without doubt outshined against the challenger from the development of timely diagnostics and prognostics to novel therapeutics and vaccinations ${ }^{1}$. The more we explore the delicate \& detailed intricacies surrounding the ongoing pandemic, there remains questions still to be answered. Rather, a reply most of the time in itself opens up newer Pandora boxes and it seems like an ongoing unlimited brawl with few troughs in between. While targeting statistical exactness among Homo Sapien's response to COVID-19 infection, we remain less educated to decide for nature's defined hostgenome variability about disease pathogenesis ${ }^{2}$. Being simplistic we all humans are the same genetically, but then we are not that same again genetically ${ }^{3}$.

2 . Our genetics aside the genetics of viral genome could have its own inherent characteristics. SARSCoV2's make \& type is also vulnerable both due to frequent genetic recombination and epigenetic stigmas by host response and possibly environment ${ }^{4}$. So every SARS-CoV2 creature will be SARS-CoV2 but again not a precise genetic clone. Like us they change behaviors as per host and the environment. Among these little entities, there must be some warriors, some just weak bystanders and possibly also having comorbids like us. So the deeper we explore, the more difficult it becomes. The response for any single human to COVID-19 can be predicted but not fine-tuned to technical requirements. Our limited understanding is based upon very preliminary data and thus possibly incomplete and sometimes faulty. While being related to medical sciences, we presume all humans and more importantly all SARS-Cov2 entities to have mathematical equivalence in terms of communicability and lethality. This inside box thought only originates once we feel exuberant in terms of information base and prone to deliver definitive statements along with imperfect prediction models ${ }^{5}$. The more we learn, the more we also decide to say "I don't know" or "I am not sure" or "I need more data".

3. While we clearly understand the dearth of matured data on the subject, we also admit the heightened awareness and medical information that has plagued this unprecedented challenge which is sometimes more of an issue than the pandemic itself 6 . Though undeniable remains the human efforts to block the viral onslaught, the losses resulting from global economic downturns, social and psychological fear vs over-confidence and unsolvable data heterogeneity still haunts us. The sociopathic "obsessive-compulsive" hand was-hing to willfully oblivious, criminal negligence are extremes we encounter on regular basis ${ }^{7}$. There are always direct questions by patients: When will the herd immunity appear after these new variants appear? When we can go unchained from masks? Will vaccine work and if yes how much? Can these variants have the potential for being more lethal? Can other viruses also go wary? From medical side there can only be half answers with definitive blanks to be filled. There goes by a day without discovering a new presentation or side effect of this virus. Available data has attempted to show its association with almost everything and anything 8,9 . The moment an information appears regardless of its evidence level it spreads faster than any other word prompting medical staff to shift policies under this information attack. The cost of changing COVID-19 specific policies appears in medicinal cost, fear, more unpredictable care provider's response and finally patient's outcome ${ }^{10}$.

4. The author firmly believes that had any other disease be pursued with this pace, stamina and political tags it must have been the "Corona Situation". This is the need of the hour to manage medical information channels and medical staff be formally taught about medical information management. Unnecessary and incomplete information sources must be filtered or replied back immediately through a concerned organization $^{11}$. Policy changes with regards to any aspect of medical management from diagnostics to vaccination must be helped through a separate department throughout the country. Standardization based upon best possible evidence from PCR kits to treatment protocols with price management needs to be taken over by governmental agencies. Not addressing such bio threats in real-time will not only disallow public calmness but patients and healthcare service providers wandering in different dimensions with each category using a different approach from the rest all adding up to additional issues along with ongoing COVID-19 pandemic ${ }^{11}$. 


\section{REFERENCES}

1. Asselah T, Durantel D, Pasmant E, Lau G, Schinazi RF. COVID-19: Discovery, diagnostics and drug develop-ment. J Hepatol 2021; 74(1): 168-84.

2. Ovsyannikova IG, Haralambieva IH, Crooke SN, Poland GA, Kennedy RB. The role of host genetics in the imm-une response to SARS-CoV-2 and COVID-19 susceptibi-lity and severity. Immunol Rev 2020; 296(1): 205-19.

3. Choudhary S, Sreenivasulu K, Mitra P, Misra S, Sharma P. Role of genetic variants and gene expression in the susceptibility and severity of COVID-19. Ann Lab Med 2021; 41(2): 129-38.

4. Chlamydas S, Papavassiliou AG, Piperi C. Epigenetic mechanisms regulating COVID-19 infection. Epigenetics 2021; 16(3): 263-70.

5. Wynants L, Van Calster B, Collins GS, Riley RD, Heinze G, Schuit E. Prediction models for diagnosis and prog-nosis of covid-19 infection: systematic review and critical appraisal. BMJ 2020; 369: m1328.

6. Tasnim S, Hossain MM, Mazumder H. Impact of Rumors and misinformation on COVID-19 in Social Media. J Prev Med Public Health 2020; 53(3): 171-74.

7. Lee SA. Coronavirus anxiety scale: A brief mental health screener for COVID-19 related anxiety. Death Stud 2020; 44(7): 393-401.

8. de Souza TH, Lanziotti VS, Lee JH. COVID-19 and chil-dren: many questions yet to be answered. Ann Acad Med Singap 2020; 49(8): 527-29.
9. Khatamzas E, Rothe C, Kroidl I. COVID-19 aus sicht der infektiologie [COVID-19: questions and answers from infectiology]. Dtsch Med Wochenschr 2020; 145(15): 1051-56.

10. Ahmed SAKS, Ajisola M, Azeem K, Bakibinga P, Chen YF, Choudhury NN. Improving Health in Slums Colla-borative. Impact of the societal response to COVID-19 on access to healthcare for non-COVID-19 health issues in slum communities of Bangladesh, Kenya, Nigeria and Pakistan: results of pre-COVID and COVID-19 lockdown stakeholder engagements. BMJ Glob Health 2020; 5(8): e003042.

11. Niemiec E. COVID-19 and misinformation: Is censorship of social media a remedy to the spread of medical misinformation? EMBO Rep 2020; 21(11): e51420.

Dr Sikandar Hayat Khan Head Department of Pathology PNS Hafeez Hospital Islamabad Pakistan

Dr Mehreen Gilani

Consultant Microbiologist

PNS Hafeez Hospital Islamabad Pakistan 